\title{
Interdisziplinäres Forschungskolloquium Protestbewegungen / Interdisciplinary research colloquium on protest movements
}

\author{
Klimke, Martin ; Scharloth, Joachim
}

\begin{abstract}
Die Protestbewegung der 1960er und 1970er Jahre werden nicht mehr allein in Erinnerungsliteratur und Feuilletons aufgearbeitet, sondern sind im vergangenen Jahrzehnt auch zum Gegenstand der historischen Wissenschaften geworden. Dies zeigt sich an der gewachsenen Zahl von Neuerscheinungen und Tagungen zum Thema. Dabei ist bemerkenswert, dass die Geschichtsschreibung von ehemaligen Aktivisten und Betroffenen immer mehr auf Wissenschaftlerinnen und Wissenschaftler übergeht, die keine Zeitzeugen waren. Von diesem Generationenwechsel ist ein neuer Blick auf diese sozialen Bewegungen zu erwarten. So arbeiten gegenwärtig zahlreiche Nachwuchswissenschaftlerinnen und -wissenschaftler aus Linguistik, Geschichtswissenschaft, Politologie, Soziologie und Literaturwissenschaft an Forschungsprojekten, die bislang vernachlässigte Aspekte der 1960er Jahre in den Blick nehmen. Das „Interdisziplinäre Forschungskolloquium Protestbewegungen“ bietet Nachwuchswissenschaftlerinnen und -wissenschaftlern ein Forum, in dem sie neue Zugänge zur Historisierung und wissenschaftlichen Behandlung der Studentenbewegung diskutieren können.
\end{abstract}

DOI: https://doi.org/10.1515/zfgl.33.2-3.343

Posted at the Zurich Open Repository and Archive, University of Zurich

ZORA URL: https://doi.org/10.5167/uzh-154584

Journal Article

Published Version

Originally published at:

Klimke, Martin; Scharloth, Joachim (2005). Interdisziplinäres Forschungskolloquium Protestbewegungen / Interdisciplinary research colloquium on protest movements. Zeitschrift für germanistische Linguistik, 33(2-3):n/a.

DOI: https://doi.org/10.1515/zfgl.33.2-3.343 


\title{
Diskussionen, Miszellen, Forschungsnotizen
}

\author{
Martin Klimke / Joachim Scharloth \\ Interdisziplinäres Forschungskolloquium \\ Protestbewegungen / International Research Forum \\ Protest. Protest Movements, Activism and Social \\ Dissent
}

Die Protestbewegung der 1960er und 1970er Jahre werden nicht mehr allein in Erinnerungsliteratur und Feuilletons aufgearbeitet, sondern sind im vergangenen Jahrzehnt auch zum Gegenstand der historischen Wissenschaften geworden. Dies zeigt sich an der gewachsenen Zahl von Neuerscheinungen und Tagungen zum Thema. Dabei ist bemerkenswert, dass die Geschichtsschreibung von ehemaligen Aktivisten und Betroffenen immer mehr auf Wissenschaftlerinnen und Wissenschaftler übergeht, die keine Zeitzeugen waren. Von diesem Generationenwechsel ist ein neuer Blick auf diese sozialen Bewegungen $\mathrm{zu}$ erwarten. So arbeiten gegenwärtig zahlreiche Nachwuchswissenschaftlerinnen und -wissenschaftler aus Linguistik, Geschichtswissenschaft, Politologie, Soziologie und Literaturwissenschaft an Forschungsprojekten, die bislang vernachlässigte Aspekte der 1960er Jahre in den Blick nehmen. Das „Interdisziplinäre Forschungskolloquium Protestbewegungen“ bietet Nachwuchswissenschaftlerinnen und -wissenschaftlern ein Forum, in dem sie neue Zugänge zur Historisierung und wissenschaftlichen Behandlung der Studentenbewegung diskutieren können.

$\mathrm{Zu}$ diesem Zweck soll auf regelmäßigen Arbeitstagungen der Austausch über Projekte und die Diskussion über Gegenstände und Methoden gefördert werden. Ziel der Tagungen ist es, Nachwuchswissenschaftlerinnen und -wissenschaftler unterschiedlicher Fachrichtungen für die vielfältigen Möglichkeiten der Perspektivierung des Forschungsgegenstandes zu sensibilisieren. Eine erste Tagung fand am 9. und 10. Juli 2004 an der Universität Heidelberg zum Thema „Gelebt, erinnert und erforscht? - 1968 auf dem Weg vom kommunikativen zum kulturellen Gedächtnis“ statt. Im Februar 2005 veranstaltete das Interdisziplinäre Forschungskolloquium am Deutschen Seminar der Universität Zürich eine Konferenz mit dem Titel „Maos Rote Garden? 1968 zwischen kulturrevolutionärem Anspruch und subversi- 
ver Praxis", in dem kultur- und mediengeschichtliche Aspekte der Studentenbewegung thematisiert wurden. Im September 2005 findet eine Tagung zum Thema "Musik und Protest" an der Evangelischen Akademie Schwerte statt. Für das Jahr 2006 ist eine Tagung zum Thema „Between the ,Prague Spring' and the ,French May': Transnational Exchange and national Recontextualization of Protest Cultures" am Heidelberg Center for American Studies an der Universität Heidelberg (25.-27. Heidelberg) geplant.

Eine wichtige Arbeitsform im Rahmen der Tagungen ist die Präsentation und Diskussion von Forschungsvorhaben und -ergebnissen. Die Tagungen dienen aber nicht ausschließlich als Forum für Projektvorstellungen. Vielmehr werden stets auch verschiedene Themenschwerpunkte behandelt. Im Themenschwerpunkt „Archiv“ werden Aufbau und Sammelschwerpunkte prominenter Archive beleuchtet. Ziel ist es, den Informationsaustausch über den Zugang zu Quellen zu befördern und praktische Hinweise über die Arbeitsbedingungen vor Ort zu geben. Von besonderem Interesse ist die Geschichte einzelner Archive, die selbst Teil der Geschichte der Studentenbewegung ist. In der Sektion "Quellen“ werden unterschiedliche Dokumententypen auf ihr heuristisches Potenzial hin und auf die methodologischen Probleme im Umgang mit ihnen befragt. Zudem soll durch den Austausch über Quellenbestände einer voreiligen Kanonisierung prominenter Ereignisse entgegen gewirkt und der Blick für den Reichtum der Ereignisse geweitet werden. Im Themenbereich „Methodologie“ wird diskutiert, welche methodischen Zugänge für bestimmte Forschungsfragen in besonderem Maße geeignet sind. Zudem soll geprüft werden, welche neuen methodischen $\mathrm{Zu}$ gänge der weiteren Erforschung der Studentenbewegung förderlich sein könnten. In der Sektion „Traditionen“ geht es um die Auseinandersetzung mit bisherigen Forschungstraditionen. Anhand der Diskussion eines Standardwerks zur Studentenbewegung soll jeweils die kritische Auseinandersetzung mit der bisherigen Forschung angeregt werden. Im Themenschwerpunkt „Disziplinen“ werden die Wege der Forschung in den einzelnen Wissenschaften in Überblicksdarstellungen referiert. Dieser Bereich fungiert als einer der maßgeblichen Impulsgeber für den interdisziplinären Austausch innerhalb des Forschungskolloquiums. Von besonderer Bedeutung ist schließlich auch eine Debatte über den gegenwärtigen gesellschaftlichen Rahmen der Erforschung der Studentenbewegung, ihrem Stellenwert in der allgemeinen öffentlichen Diskussion sowie ihre (Selbst-)Verortung innerhalb vergangener und gegenwärtiger Protestbewegungen.

Dem Forumscharakter trägt das Interdisziplinäre Forschungskolloquium auch insofern Rechnung, als es Wissenschaftlerinnen und Wissenschaftlern, die sich mit Protestbewegungen beschäftigen, die Möglichkeit gibt, ihre Projekte auf einer Internetplattform zu präsentieren. Die Webseite (www.ifk-protestbewegungen.org) dient der Orientierung, welche Themen 
bereits wissenschaftlich behandelt werden, und soll den Austausch unter den Nachwuchswissenschaftlern fördern.

Das Interdisziplinären Forschungskolloquium wird von Martin Klimke (Universität Heidelberg) und Joachim Scharloth (Universität Zürich) geleitet und zählt mehr als 40 Affiliierte aus zahlreichen europäischen Ländern und den USA. Es steht allen Nachwuchswissenschaftlerinnen und -wissenschaftlern offen, die sich mit den Protestbewegungen des 20. und 21. Jahrhunderts beschäftigen.

\author{
Adressen der Verfasser: \\ Martin Klimke, Historisches Seminar, Universität Heidelberg, Grabengasse 3-5, D-69117 \\ Heidelberg. \\ E-mail:martin.klimke@uni-hd.de \\ Dr. Joachim Scharloth, Deutsches Seminar, Universität Zürich, Schönberggasse 9, CH-8001 \\ Zürich. \\ E-mail:scharloth@access.unizh.ch
}

